\title{
Intérêt d'une réorganisation des services de chirurgie digestive en période de pandémie COVID -19
}

Interest of reorganizing digestive surgery services during a COVID-19 pandemic

\author{
Merouane BOUKRISSA ${ }^{1,3}$, Khadidja BRAHMI ${ }^{2,3}$ \\ 1 Service des Urgences Chirurgicales, CHU Oran \\ ${ }^{2}$ Clinique Chirurgicale A, CHU Oran \\ ${ }^{3}$ Faculté de Médecine d'Oran, Algérie
}

Auteur correspondant : mboukrissa@hotmail.com

Article : Stratégie pour la pratique de la chirurgie digestive et oncologique en situation d'épidémie de COVID-19 avoirs expérientiels et normes collectives d'automédication

Author links open overlay panelJ.-J.TuechabA.GangloffcF.DiFiorebcP.MichelbcC.BriganddK.SlimeM. PocardfgL.Schwarzab Show more https://doi.org/10.1016/j.jchirv.2020.03.007Get rights and content

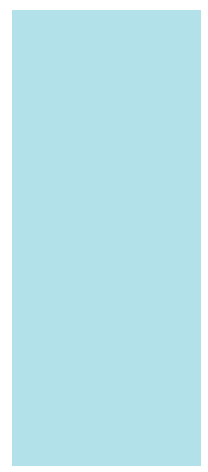

La crise pandémique liée au COVID-19 impose une réorganisation des circuits de prise en charge de la chirurgie digestive. La chirurgie d'urgence reste prioritaire, en revanche, les autres chirurgies non urgentes " dites fonctionnelles " seront reportées dans le but d'accroitre les capacités d'hospitalisation conventionnelle et en soins intensifs. Cependant, va se poser la question du timing de la prise en charge chirurgicale des cancers digestifs, du report ou non ? Faut-il opérer coute que coute, au prix d'un sur risque lié à la pandémie, évitable, pouvant être fatal pour l'opéré.

L'état d'immunodépression liée au cancer même, à la chimiothérapie, à la chirurgie et à l'anesthésie rend les patients atteints de maladie cancéreuse plus exposés au risque d'infection COVID-19. En outre, selon une étude chinoise récente, ces patients sont à plus haut risque de développer des épisodes sévères (39\% vs 8 \%)[1]. Lorsque l'intervention chirurgicale ou une cure de chimiothérapie avait été observée dans le mois précédant l'apparition du virus, le risque de développer un épisode sévère était de $75 \%$ contre $43 \%$ pour les patients n'ayant pas été opérés ou n'ayant pas reçu de chimiothérapie au cours de cette période. L'impact oncologique d'un report de prise en 
charge chirurgicale est mis en balance avec le sur risque de mortalité en cas d'infection respiratoire grave due au coronavirus.

Ainsi des stratégies ont été proposées de surseoir au traitement chirurgical pour les cancers coliques (T1-2, N0) ; de recommander une chimiothérapie néo adjuvante et d'attendre pour les atteintes tumorales coliques avancées. Une radiochimiothérapie pour les cancers du rectum T3-4 et/ ou $\mathrm{N}+$, ou discussion en réunion de concertation pluridisciplinaire (RCP) pour une radiothérapie courte dans le but de réduire le temps d'exposition infectieuse à l'hôpital. De même, qu'il est recommandé de reporter la majorité des chirurgies œsogastrique, hépatique ou pancréatique en raison de leur forte morbi-mortalité.

Des équipes chinoises ont publié plusieurs articles suggérant de modifier les pratiques et d'adapter les stratégies. Il existe des points communs à la prise en charge des patients cancéreux : lutter contre la dénutrition en apportant des compléments alimentaires ou par la mise en place d'une alimentation entérale par sonde d'alimentation ; éviter une altération importante du système immunitaire par des traitements agressifs ; éviter les hospitalisations, les visites, les séjours à l'hôpital qui favoriseraient la contamination par le virus; privilégier une séquence thérapeutique qui n'impose pas un timing chirurgical strict qui ne pourra peut-être pas être réalisé ; discuter des prises en charge en RCP afin de définir le projet thérapeutique el plus adapté au patient et à la période d'épidémie. Ces réunions devront se faire de façon virtuelle (RCP virtuelle) ; une aide psychologique sera nécessaire aux patients qui doivent faire face à la gestion du cancer et à la gestion de l'épidémie [2], [3], [4], [5].

Autre situation, en cas d'indication opératoire urgente d'un patient infecté ou suspect d'infection par le COVID-19, des mesures de protection stricte du personnel soignant doivent être entreprises par une communication large avec l'équipe du bloc opératoire et une salle opératoire en dépression si possible avec minimisation de l'impact respiratoire de l'intervention. Le choix de la voie d'abord devra être discuté au cas par cas avec l'anesthésiste. la laparoscopie étant à privilégier si la pathologie causale le permet et si l'état cardio respiratoire est stable; dans les autres cas, une laparotomie reste indiquée.

En période postopératoire, le secteur d'hospitalisation doit être adapté à l'état respiratoire de l'opéré. L'aménagement en chambres individuelles sera souhaitable afin de limiter les infections croisées avec d'autres patients ou des membres de la famille.

\section{Références bibliographiques}

[1]. W. Liang, W. Guan, R. Chen, et al.Cancer patients in SARS-CoV-2 infection: a nationwide analysis in China Lancet Oncol, 21 (3) (2020), pp. 335-337 ArticleDownload PDFView Record in ScopusGoogle Scholar

[2]. Y. Zhang, J.M. XuMedical diagnosis and treatment strategies for malignant tumors of the digestive system during the outbreak of novel coronavirus pneumonia Zhonghua Zhong Liu Za Zhi, 42 (0) (2020), p. E005 View Record in ScopusGoogle Scholar

[3]. Y.H. Chen, J.S. PengTreatment strategy for gastrointestinal tumor under the outbreak of novel coronavirus pneumonia in China Zhonghua Wei Chang Wai Ke Za Zhi, 23 (2) (2020), pp. I-IV View Record in ScopusGoogle Scholar

[4]. X.H. Hu, W.B. Niu, J.F. Zhang, et al.Thinking of treatment strategies for colorectal cancer patients in tumor hospitals under the background of coronavirus pneumonia Zhonghua Wei Chang Wai Ke Za Zhi, 23 (3) (2020), p. E002 View Record in ScopusGoogle Scholar

[5]. F. Wu, Y. Song, H.Y. Zeng, et al.Discussion on diagnosis and treatment of hepatobiliary malignancies during the outbreak of novel coronavirus pneumonia Zhonghua Zhong Liu Za Zhi, 42 (0) (2020), p. E004 View Record in Scopus Google Scholar 